\title{
Эффекты последействия в методе переходных процессов: индукционно-вызванная поляризация
}

Кожевников Н. О., Антонов Е. Ю.

\begin{abstract}
Аннотация
Одним из эффектов последействия, присущих геологической среде и оказывающих влияние на результаты метода переходных процессов, является индукционно-вызванная электрическая поляризация (ВПИ). Ее влияние на индукционные переходные характеристики выражается в нарушении монотонности спада ЭДС (включая изменение полярности). Зависимость ВПИ от многих факторов затрудняет изучение основных закономерностей ее проявления. Одним из путей решения этой проблемы является представление результатов моделирования с помощью нормированных переходных характеристик. C наиболее общих позиций фактором, определяющим интенсивность и временной диапазон проявления ВПИ, является конкуренция между процессами индукции и вызванной поляризации. Процессы вызванной поляризации протекают по-разному в зависимости от того, какой источник используется для их возбуждения. Поэтому при изучении поляризующихся сред соответствие между результатами метода переходных процессов и традиционного метода ВП наблюдается не всегда.
\end{abstract}

\section{Ключевые слова:}

Индукционно-вызванная поляризация, метод переходных процессов 


\title{
ЭФФЕКТЫ ПОСЛЕДЕЙСТВИЯ В МЕТОДЕ ПЕРЕХОДНЫХ ПРОЦЕССОВ: ИНДУКЦОННО-ВЫЗВАННАЯ ПОЛЯРИЗАЦИЯ
}

\author{
Н. О. Кожевников, Е. Ю. Антонов \\ Институт нефтегазовой геологии и геофизики СО РАН
}

Одним из эффектов последействия, присущих геологической среде и оказывающих влияние на результаты метода переходных процессов, является индукционно-вызванная электрическая поляризация (ВПИ). Ее влияние на индукционные переходные характеристики выражается в нарушении монотонности спада ЭДС (включая изменение полярности). Зависимость ВПИ от многих факторов затрудняет изучение основных закономерностей ее проявления. Одним из путей решения этой проблемы является представление результатов моделирования с помощью нормированных переходных характеристик. С наиболее общих позиций фактором, определяющим интенсивность и временной диапазон проявления ВПИ, является конкуренция между процессами индукции и вызванной поляризации. Процессы вызванной поляризации протекают по-разному в зависимости от того, какой источник используется для их возбуждения. Поэтому при изучении поляризующихся сред соответствие между результатами метода переходных процессов и традиционного метода ВП наблюдается не всегда.

Индукционно-вызванная поляризация, метод переходных процессов

\section{Введение}

Основными параметрами геологической среды, изучаемыми методами электроразведки, является удельное электрическое сопротивление $\rho$ или удельная электропроводность $\sigma=1 / \rho$. В большинстве случаев предполагается, что $\sigma$ или $\rho$ не зависят от времени/частоты. В теории низкочастотных и/или импульсных индуктивных методов принимается также, что магнитная проницаемость $\mu$ геологической среды не зависит от времени или частоты. Такой подход основан на предположении, что эффекты последействия в геологической среде отсутствуют. Однако нередко эти эффекты проявляются в виде вызванной электрической поляризации (ВП) и релаксации намагниченности, или магнитной вязкости (MB).

Обычно для изучения вызванной поляризации применяются установки с заземленными питающей и приемной линиями. Однако вызванная поляризация проявляется и при индуктивном возбуждении поля, особенно в методе переходных процессов. Что касается эффектов магнитной вязкости, то они сильнее всего проявляются именно в методе переходных процессов.

Изучению процессов ВП, наблюдающихся при работе установками с заземлёнными линиями, посвящена обширная литература. Методика и результаты лабораторных измерений магнитной вязкости на образцах также отражены во многих публикациях. Вместе с тем все еще не хватает работ, в которых дается анализ и обобщение эффектов ВП и МВ в методе переходных процессов.

Даная статья, которая обобщает результаты изучения авторами основных закономерностей проявления вызванной поляризации в методе переходных процессов, до некоторой степени восполняет этот пробел. Статья построена следующим образом. Сначала приводится описание модели Коул-Коул, которая использовалась для расчета индукционных переходных характеристик с учетом вызванной поляризации. Затем на примере слабо поляризующейся среды мы рассматриваем, как возникает индукционно вызванная поляризация. После этого мы приводим определение нормированной переходной характеристики и, воспользовавшись 
такой формой [способом] представления данных, на примере однородного поляризующегося полупространства и двухслойной среды с поляризующимся верхним слоем показываем, как эффект ВПИ проявляется в зависимости от параметров модели Коул-Коул, геоэлектрического разреза и установки. Далее мы описываем подход, с помощью которого удается получить объективную оценку достоверности результатов инверсии индукционных переходных характеристик с учетом ВПИ. В последнем разделе статьи мы описываем отличия ВПИ от вызванной поляризации, наблюдаемой с заземленными линиями. В конце кратко сформулированы основные выводы.

Представленные в статье результаты получены с помощью оригинальных программ. Алгоритмы, положенные в их основу, а также сами программы подробно описаны в [Антонов и др., 2014; Эпов, Антонов, 1999, 2000].

\section{Электропроводность геологических сред с учётом вызванной поляризации}

В настоящее время расчёт и интерпретация переходных характеристик горизонтальнослоистых сред с учётом ВПИ стали рутинной процедурой [Антонов и др., 2014; Seidel, Tezkan, 2017]. Кроме этого, уже появились программы, позволяющие моделировать и выполнять инверсию индукционных переходных характеристик для трёхмерных поляризующихся сред. В литературе приводятся примеры использования этих программ при решении прямой и обратной задач метода переходных процессов [Marchant et al., 2014; Kang, 2017].

Чаще всего при решении прямой и обратной задач МПП отклик среды рассчитывают в частотной области, после чего преобразуют его во временную область. Учёт вызванной поляризации осуществляется путем использования комплексного, зависящего от частоты удельного электрического сопротивления $\rho^{*}(\omega)$, описываемой формулой Коул-Коул [Pelton, 1978; Flis et al., 1989]:

$$
\rho^{*}(\omega)=\rho_{0}^{\square} 1-m_{\square}^{\square} 1-\frac{1}{1+(j \omega \tau)^{c}} \square,
$$

где $j=\sqrt{-1} ; \omega-$ круговая частота, рад/с; $\rho_{0}$ - удельное электрическое сопротивление на постоянном токе, Ом · $; ~ m-$ поляризуемость, $c$ - показатель степени; $\tau$ - время релаксации, с. Диапазон возможных значений параметра $m-$ от нуля до единицы, $\tau$ - от нуля до бесконечности. Параметр с может изменяться в пределах от 1 (одно время релаксации) до 0 (бесконечно широкое равномерное распределение времен релаксации).

Поляризуемость $m$ можно выразить в виде [Sumner, 1976]:

$m=\frac{\rho_{0}-\rho_{\infty}}{\rho_{0}}=\frac{\Delta \rho}{\rho_{0}}$,

где $\rho_{\infty}-$ удельное электрическое сопротивление на частоте, значительно превышающей частоту релаксации $\omega_{\theta}=1 / \tau$.

\section{Индукционно-вызванная поляризация}

Впервые вызванная электрическая поляризация горных пород наблюдалась К. Шлюмберже в 1913 г. [Seigel et al., 2007]. C тех пор метод ВП нашел применение при решении задач рудной и нефтяной геологии, гидрогеологии, а в последние 25 лет - геоэкологии и геокриологии. В этом методе возбуждение и измерение вызванной поляризации осуществляется с помощью заземлённых линий; при этом воздействием на среду является ток в питающей линии, а 
откликом - разность потенциалов между приёмными электродами. В частотной области на одной либо нескольких частотах измеряют модуль и/или фазу напряжения между приёмными электродами, либо - в методе ИНФАЗ ВП - разность фаз на двух частотах [Куликов, Шемякин, 1978]. При изучении вызванной поляризации во временной области в землю подается постоянный ток, который в некоторый момент выключается, после чего производится измерение спадающего во времени напряжения - переходная характеристика ВП [Sumner, 1976; Комаров, 1978].

В методе переходных процессов при выключении установившегося тока в генераторной петле в окружающем пространстве индуцируется электрическое поле, под действием которого в земле возникают вихревые токи. Их распространение по вертикали и горизонтали контролируется распределением удельной электропроводности. В поляризующейся среде появляется «добавка», известная как индукционно-вызванная поляризация, или ВПИ [5, 6]. Измерив магнитное поле устанавливающихся токов или скорость его изменения, можно в рамках принятой модели, например, Коул-Коул, оценить поляризационные параметры геологической среды.

Нагляднее всего возникновение ВПИ можно проиллюстрировать на примере слабо поляризующейся проводящей среды, т. е. такой, для которой $\Delta \rho<<\rho_{0}$ [Flis et al., 1989; Kozhevnikov, Antonov, 2012]. Предположим, что на поверхности проводящей неполяризующейся среды расположена петля, по которой протекает установившийся ток. В момент $t=0$ ток мгновенно выключается, при этом первичное магнитное поле $\boldsymbol{B}_{1}$ петли также мгновенно убывает от установившегося значения до нуля. В этот момент в подстилающей петлю геологической среде возникают вихревые токи, создающие вторичное магнитное поле $\boldsymbol{B}_{2}$, которое на ранних временах не отличается от магнитного поля, существовавшего в среде до выключения тока в петле.

Вихревые токи представляют собой свободные заряды (ионы), перемещающиеся под действием вихревого электрического поля $\boldsymbol{E}_{\text {вихр }}$, которое контролируется сопротивлением $\rho$ : $\boldsymbol{E}_{\text {вихр }}=\boldsymbol{j}_{\text {вихр }} \rho$.

Теперь предположим, что геологическая среда содержит области или включения, в которых имеются связанные заряды, т.е. способна поляризоваться. Поскольку поляризуемость

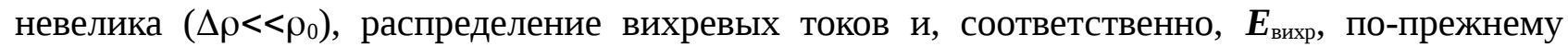

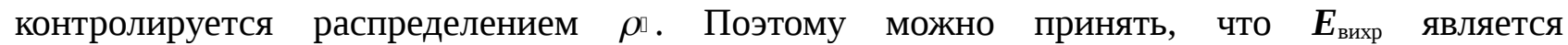
воздействием, а поляризация включений со связанными зарядами - откликом на него.

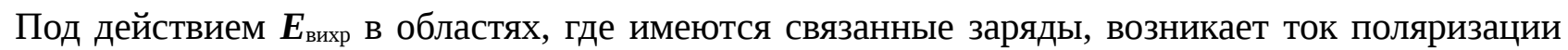

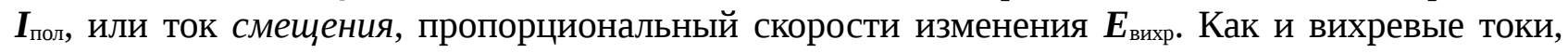
токи поляризации также создают магнитное поле, которое вносит вклад в суммарную переходную характеристику петли.

Сначала поляризационные токи имеют такое же направление, как и вихревые. В какой-то момент времени дальнейшее разделение связанных зарядов становится невозможным, и процесс поляризации заканчивается. Когда вследствие тепловых потерь интенсивность вихревых токов уменьшается на много порядков, внешнее воздействие на связанные заряды исчезает, и они возвращаются к равновесному распределению, т. е. возникает ток поляризации, протекающий в направлении, противоположном по отношению $\mathrm{K}$ первоначальному. На этой стадии магнитное поле, создаваемое токами поляризации, имеет полярность, противоположную по отношению к создаваемому «нормальными» вихревыми токами. 
В зависимости от параметров геоэлектрической модели, размеров генераторной петли и положения приемной петли наблюдаются переходные процессы с однократной или двукратной сменой полярности ЭДС, а также немонотонные процессы без изменения полярности [Левченко, 1992; Сидоров, 1985; Kamenetsky et al., 2014; Raiche, 1983; Smith, Klein , 1996; Spies, 1980; Walker, Kawasaki, 1988; Weidelt, 1982]. В качестве примера на рис. 1 приведены индукционные переходные характеристики для трех соседних пунктов зондирования на профиле, располагавшемся на расстоянии 10 км к северо-западу от трубки «Нюрбинская», Западная Якутия [Kozhevnikov, Antonov, 2006]. Для измерения использовалась аппаратура Цикл-микро и соосная установка с генераторной петлей размером 200м × 200м; размер приёмной петли составлял 100м × 100м.

\section{Оценка проявления индукционно-вызванной поляризации}

Обсуждению результатов расчётов и интерпретации индукционных переходных характеристик с учётом ВПИ посвящена обширная литература. Однако, как отмечалось выше, общие закономерности проявления ВПИ в зависимости от параметров геологической среды и установки, даже применительно к простым моделям, остаются недостаточно изученными.

В значительной степени [мере] это связано с тем, что в зависимости как от типа и размеров генераторно-приёмной установки, так и параметров геологической среды переходные процессы, даже не осложнённые влиянием ВП, по уровню и временному диапазону отличаются на несколько, а иногда и на много порядков. Кроме того, установка и геологическая среда влияют на форму индукционных переходных характеристик. В случае же поляризующейся среды число параметров, которые влияют на переходную характеристику, возрастает, что затрудняет анализ и особенно обобщение результатов моделирования.

В своих исследованиях мы ориентировались на оценку проявлений ВПИ не в абсолютном, а в относительном выражении. Это позволило отобразить данные моделирования эффектов ВПИ, в компактном, доступном для анализа и обобщения виде.

Для анализа и сопоставления результатов моделирования мы вместо ЭДС $V_{\text {вп }}(t)$ на зажимах приёмной петли, расположенной на поверхности поляризующейся среды, использовали нормированную переходную характеристику $Y(t)$. Она вычисляется по формуле [Kozhevnikov, Antonov, 2006; Кожевников, Антонов, 2020]:

$$
Y(t)=\frac{V_{B \Pi}(t)}{V_{0}(t)}
$$

где $V_{0}(t)$ - ЭДС переходного процесса, измеренная на поверхности референтной среды,

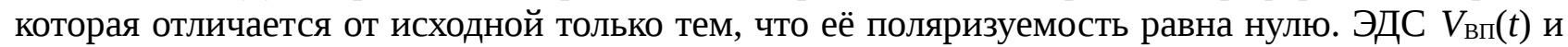
$V_{0}(t)$ в формуле (3) рассчитываются при одинаковом токе в генераторной петле (обычно 1A). Очевидно, чем больше $Y(t)$ отличается от единицы, тем сильнее - в относительном выражении - сказывается влияние ВПИ на времени $t$.

Для того, чтобы проиллюстрировать этот подход, рассмотрим, как удельное электрическое сопротивление $\rho_{0}$ поляризующегося полупространства влияет на индукционные переходные характеристики. Для простоты далее мы не используем нижний индекс, т. е. обозначаем это удельное сопротивление как $\rho$.

На рис. 2а показаны переходные характеристики для совмещённой установки размером $100 \times 100 \mathrm{M}$, расположенной на поверхности поляризующегося $(m=0.1, \tau=50$ мкс, $c=1)$ 
полупространства с сопротивлением $\rho=10,10^{2}$ и $10^{3}$ Ом · м. Такие параметры полупространства выбраны на том основании, что они являются типичными для мерзлых пород, распространенных в северных районах, где проявления ВПИ носят массовый характер [Стогний, 2008; Kozhevnikov, Antonov, 2006, 2012].

Для сравнения на рис. 2а приведены переходные характеристики для неполяризующегося полупространства. Как видно, когда $\rho=10$ Ом·м, эффекты ВПИ практически не проявляются. При $\rho=10^{2}$ Ом $\cdot$ м зависимость $V(t) / I$ становится немонотонной. При дальнейшем увеличении $\rho\left(10^{3}\right.$ Ом·м) в окрестностях временной задержки $t \approx 100$ мкс наблюдается двойная смена знака полярности ЭДС.

В отличие от графиков на рис. 2а нормированные переходные характеристики $Y(t)$ при любом сопротивлении и во всем временном диапазоне наглядно отображают проявление ВПИ (рис. 2б). На ранних временах влияние поляризуемости приводит к повышению, а на поздних - понижению ЭДС, индуцируемой в приёмной петле. Соответственно, на графиках $Y(t)$ наблюдаются максимум и минимум.

Переходные характеристики, показанные на рис. 2a, рассчитаны при $c=1$. Однако в общем случае показатель степени может быть другим. Как показали расчеты, в области ранних времен на графиках $Y(t)$ всегда наблюдается максимум. На поздних временах вид графиков зависит от показателя степени $c$ формулы Коул-Коул: когда $c<1 / 2, Y(t)$ неуклонно [монотонно] убывает; если же $c>1 / 2$, на графиках $Y(t)$ наблюдается минимум.

Как показано в [Кожевников, Антонов, 2020], в большинстве случаев амплитуда минимума $A_{\min }=1-Y_{\min }$ превышает амплитуду максимума $A_{\max }=Y_{\max }-1$. По сравнению с максимумом минимум наблюдается в более широком временном диапазоне, причем тогда, когда собственная переходная характеристика измерительной системы уже не влияет результаты измерений. Поэтому мы использовали преимущественно координаты минимума $\left(t_{\min }, Y_{\min }\right)$ нормированной переходной характеристики, которые, как выяснилось, являются информативными и удобными параметрами для интегральной оценки проявления ВПИ. Единственным ограничением на использование этих параметров является необходимость выполнения условия: $c>1 / 2$.

В упомянутой работе [Кожевников, Антонов, 2020] подробно рассмотрено, как параметры модели Коул-Коул и размеры установки влияют на нормированные переходные характеристики однородного проводящего и поляризующегося полупространства (в первую очередь на координаты минимума $\left.t_{\min }, Y_{\min }\right)$.

Результаты моделирования для однородного поляризующегося полупространства обобщены в таблице 1. Знак «+» означает, что возрастание соответствующего параметра из левого столбца таблицы приводит к увеличению $t_{\min }, Y_{\min }$. Знак «-» показывает, что $t_{\min }$ или $Y_{\min }$ уменьшается. Символ в крайнем правом столбце таблицы показывает влияние соответствующего параметра на ширину временного диапазона, в котором проявляется ВПИ.

Тот факт, что интенсивность проявления ВПИ зависит не только от поляризуемости, но и от остальных параметров $\left(\rho_{0}, \tau, c\right)$, а также размеров установки, объясняется конкуренцией между процессами индукции и вызванной поляризации. Изменение параметров модели КоулКоул и/или размеров установки сдвигает баланс в ту или иную сторону и тем самым оказывает влияние на проявление ВПИ. В этом заключается одно из отличий между ВПИ и процессами вызванной поляризации, изучаемыми в традиционном методе ВП. 
Особенно наглядно зависимость интенсивности ВПИ от соотношения между индукционными и поляризационными процессами можно проиллюстрировать на примере проводящего поляризующегося слоя, подстилаемого слабо проводящим [высокоомным] неполяризующимся основанием. Применительно к изучению криолитозоны эта модель описывает слой мёрзлых пород, подстилаемых талыми. При значительной толщине слоя такая модель аппроксимирует геоэлектрический разрез криолитозоны в целом: сверху мощная толща мёрзлых пород, внизу - талое основание. При небольшой толщине слоя эта модель соответствует разрезам с сезонно-мёрзлым слоем.

Рассмотрим, как интенсивность проявления ВПИ изменяется в зависимости от мощности $h_{1}$ и удельного электрического ссопротивления $\rho_{1}$ слоя. Как и в рассмотренном выше случае однородного полупространства, расчёты выполнены для установки с совмещёнными петлями размером $100 \mathrm{M} \times 100 \mathrm{M}$.

Рис. За иллюстрирует зависимость $Y_{\min }$ от мощности $h_{1}$ поляризующегося слоя. При расчётах было принято, что УЭС слоя $\rho_{1}$ равно 100 Ом $\cdot$ м, а его мощность изменяется от 0.1 м до 1000 м. Остальные параметры слоя: $m_{1}=0.1, \tau_{1}=50$ мкс, $c_{1}=1$. Графики $Y_{\min }\left(h_{1}\right)$, показанные на рис. За рассчитаны для УЭС неполяризующегося основания в диапазоне от 10 до $10^{4} \mathrm{OM} \cdot \mathrm{M}$.

Как видно на рис. За (графики 1 и 2), когда $\rho_{2} \leq \rho_{1}$, увеличение $h_{1}$ приводит к монотонному убыванию $Y_{\min }$ от одного асимптотического значения (1 при $\left.h_{1}=0\right)$ до другого $(0.7$ при $\left.h_{1} \geq 100 \mathrm{M}\right)$. Если же $\rho_{2}>\rho_{1}$ (графики 3-5), наблюдается другая картина: по мере возрастания $h_{1}$ $Y_{\min }$ убывает, достигает отчетливо выраженного минимума, а затем возрастает, асимптотически приближаясь к значению, наблюдаемому в присутствии поляризующегося полупространства с сопротивлением $\rho_{1}=100$ Ом $\cdot$ м. Таким образом, если $\rho_{2}>\rho_{1}$ (слабо проводящее основание), эффект ВПИ выражен намного сильнее, чем в тех случаях, когда $\rho_{2} \leq \rho_{1}$. Для модели со слабо проводящим основанием увеличение $\rho_{2}$ приводит к тому, что минимум на графике $Y_{\min }\left(h_{1}\right)$ становится все более глубоким. Так, $Y_{\min }=-0.94$ при $\rho_{2}=10^{3} \mathrm{Oм} \cdot \mathrm{m}$ (график 3), тогда как при $\rho_{2}=10^{4} \mathrm{OM} \cdot \mathrm{m} Y_{\min }=-18$ (график 5).

Таким образом, эффект ВПИ от тонкого поляризующегося слоя выражен намного сильнее, чем от слоя большой мощности или даже поляризующегося полупространства. Отметим другие особенности графиков $\left.Y_{\min }\left(h_{1}\right): 1\right)$ при некоторой мощности слоя наблюдается максимальный эффект ВПИ; 2) одно и то же значение $Y_{\min }$ может наблюдаться при двух разных $h_{1}$.

Неочевидное, на первый взгляд, проявление ВПИ для рассматриваемой модели объясняется конкуренцией между вихревыми токами и токами поляризации. В тонком слое «обычные» вихревые токи быстро затухают, что создает благоприятные условия для проявления ВПИ [Smith et al., 1988]. С другой стороны, поскольку слой тонкий, объём поляризующегося материала невелик, и проявления ВПИ (в абсолютном выражении) незначительны. При увеличении мощности слоя вклад ВПИ в суммарный сигнал возрастает, при этом «с точки зрения» индукционных эффектов слой все ещё остается тонким, и вихревые токи попрежнему быстро затухают. При некоторой мощности слоя наблюдается оптимальное соотношение между поляризационными и вихревыми токами, при котором эффекты ВПИ в относительном выражении вносят максимальный вклад в результирующую переходную характеристику. Дальнейшее увеличение мощности слоя приводит к преобладанию индукционных эффектов, и влияние ВПИ снижается. Поэтому в случае тонкого поляризующегося слоя, залегающего на высокоомном основании, эффект ВПИ проявлен более сильно, чем в случае слоя большой мощности или даже полупространства с такими же параметрами $(\rho, m, \tau, c)$, как у слоя. 
Влияние удельного электрического сопротивления слоя на $Y_{\min }$ иллюстрирует рис. Зб. При расчетах было принято, что $\rho_{1}$ изменяется от 10 до $10^{4} \mathrm{OM} \cdot \mathrm{M}$, а остальные параметры слоя такие же, как в рассмотренном выше случае (см. рис За). Слой залегает на высокоомном $\left(\rho_{2}=10^{4} \mathrm{OM} \cdot \mathrm{M}\right)$ основании. Графики зависимости $Y_{\min }\left(\rho_{1}\right)$ на рис. Зб приведены для слоя мощностью 1,10 и 100м. На графиках $Y_{\min }\left(\rho_{1}\right)$ наблюдается минимум. При фиксированной мощности слоя максимальный эффект ВПИ наблюдается при некотором значении удельного сопротивления слоя, которое тем меньше, чем тоньше слой.

Комментируя рис. Зб, отметим следующий факт: чем меньше $h_{1}$, тем более глубоким становится минимум на графике $Y_{\min }\left(\rho_{1}\right)$. Таким образом, при уменьшении мощности слоя проявление ВПИ становится более интенсивным. Интересно, что при любой мощности слоя минимум на графиках $Y_{\min }\left(\rho_{1}\right)$ наблюдается, когда проводимость слоя $S_{1}=h_{1} / \rho_{1}$ составляет 0.045 См. Очевидно, в рассматриваемом случае параметром, контролирующим максимальный эффект слоя, является его проводимость. Как и графики $Y_{\min }\left(h_{1}\right)$ (см. рис. 4a), графики $Y_{\min }\left(\rho_{1}\right)$ на рис. 4б отражают тот факт, что параметры слоя (в данном случае, удельное сопротивление) влияют на соотношение между вкладами индукционного и поляризационного эффектов в результирующую переходную характеристику.

\section{Инверсия индукционных переходных характеристик с учётом вызванной поляризации}

На сегодняшний день опубликовано много работ, в которых представлены и обсуждаются результаты инверсии индукционных переходных характеристик с учетом ВПИ [Кожевников и др., 2014; Компаниец и др., 2013; Kang et al., 2017; Marchant et al., 2014; Seidel, Tezkan, 2017]. Эти результаты иллюстрируют несомненные успехи, достигнутые в последние годы в интерпретации данных наземных и аэросъемок методом переходных процессов при изучении поляризующихся сред, в том числе трехмерных. Вместе с тем, на наш взгляд, в этих работах не уделяется должного внимания оценке достоверности результатов решения обратной задачи. Поэтому мы ограничиваемся изложением подхода, который, как нам представляется, может оказаться полезным в тех случаях, когда требуется составить объективное представление о том, в какой мере геоэлектрические модели, найденные путем инверсии, отражают реальность [Кожевников, Антонов, 2009, 2010; Kozhevnikov, Antonov, 2008].

При инверсии индукционных переходных характеристик поляризующейся среды число подлежащих определению параметров возрастает по сравнению с тем, которое необходимо для описания неполяризующейся среды. В случае многослойной модели число параметров оказывается настолько большим, что неизбежно возникают проблемы с анализом, структурированием и обобщением результатов инверсии. Поэтому мы сосредоточились на оценке возможностей инверсии при определении параметров простых и вместе с тем фундаментальных моделей, а именно однородного поляризующегося полупространства и двухслойных горизонтально-слоистых сред, в которых поляризующимся является верхний слой либо основание.

В настоящее время не существует природных объектов, электрические свойства которых изучены настолько детально, чтобы на основе экспериментальных данных можно было оценить качество инверсии данных МПП с учетом ВПИ. В своих исследованиях мы использовали синтетические данные, однако компьютерные эксперименты были задуманы и проводились так, чтобы по возможности имитировать «реальность». Один из соавторов настоящей статьи (\#1) составлял наборы моделей, рассчитывал для них индукционные переходные характеристики и добавлял к ним шум с параметрами, близкими к наблюдаемым на практике. Другой соавтор (\#1) выполнял инверсию этих данных в рамках модели горизонтально-слоистой проводящей поляризующейся среды. 
Сначала соавтор-интерпретатор выполнял инверсию, ничего не зная о моделях. После этого ему сообщалась некоторая информация, и он снова проводил инверсию, на этот раз с учётом априорной информации. Если возникала необходимость, первый соавтор сообщал дополнительные сведения, которые учитывались на следующем этапе инверсии. Наконец, найденные в результате инверсии модели сравнивались с исходными. Это позволяло на каждом из этапов объективно оценивать качество решения обратной задачи применительно к конкретной модели.

Проиллюстрируем этот подход на примере двухслойной среды с поляризующимся верхним слоем. Параметры исходных моделей приведены в таблице 2.

Для того чтобы выяснить, как результаты инверсии зависят от геометрии установки, а также оценить возможности совместной инверсии данных МПП, измеренных установками разной геометрии, переходные характеристики рассчитывались для двух установок - «петля в петле» и совмещенной. В первом случае размер генераторной петли составлял $200 \mathrm{M} \times 200 \mathrm{M}$, приемной $-50 \mathrm{M} \times 50 \mathrm{~m}$, во втором - обе петли имели размер $50 \mathrm{M} \times 50$ м. Для имитации внешних электромагнитных помех и шумов аппаратуры на синтетические переходные характеристики накладывался шум, полученный путем генерации последовательностей нормальнораспределенных случайных чисел.

Диапазон времён, в котором были рассчитаны псевдо-экспериментальные переходные характеристики, определялся размерами установки, помехами и возможностями аппаратуры. Практика полевых работ показывает, что собственные переходные процессы в установках $50 \mathrm{M} \times 50$ м и 200м $\times 50$ м заканчиваются на временах порядка 10мкс и 30мкс, соответственно. Конечное время «регистрации» для псевдо-экспериментальных переходных характеристик составляло 100 мс, однако для инверсии использовались отсчеты ЭДС только на тех задержках, где сигнал по порядку величины превышал 0.1 мкВ.

Интерпретатору были переданы переходные характеристики, рассчитанные для модели с неполяризующимся основанием. Приступая к их инверсии, он ничего не знал о моделях. По внешнему виду переходных характеристик методом «проб и ошибок» автор\#2 пришел к выводу, что инверсия может быть выполнена в рамках двухслойной модели. Сначала были подобраны модели для совмещенной установки, затем - для установки «петля в петле», после чего проведена совместная инверсия данных для обеих установок. В результате как индивидуальной, так и совместной инверсии все 5 моделей были идентифицированы как модели с поляризующимся верхним слоем и неполяризующимся основанием.

Для отображения и обобщения данных, полученных в результате инверсии, мы использовали способ, заключающийся в построении графиков, на которых по оси абсцисс откладывается один из параметров формулы Коул-Коул, принятый в качестве независимого (например, поляризуемость $m$ ), а по оси ординат - параметр, определенный в результате инверсии [Кожевников, Антонов, 2007]. Такой подход позволил наглядно показать, как при изменении того или иного параметра меняется качество подбора этого же или других параметров. Для облегчения восприятия и анализа графиков по оси ординат откладывались нормированные значения найденных в результате инверсии параметров, например $m_{\mathrm{inv}} / m$, где $m$ - «истинная», т. е. использовавшаяся при расчёте переходных характеристик поляризуемость, $m_{\text {inv }}-$ поляризуемость, найденная в результате инверсии.

В рассматриваемом случае в качестве независимого параметра логично было использовать мощность $h_{1}$ слоя (рис. 4). Для облегчения восприятия и анализа графиков по оси ординат откладываются нормированные значения найденных в результате инверсии параметров. На 
рис. $4 h_{1}, \rho_{1}, m_{1}, \tau_{1}, c_{1}$ и $\rho_{2}$ обозначают «истинные», т.е. принятые при расчете псевдоэкспериментальных переходных характеристик параметры модели, а $h_{1 \text { inv }}, \rho_{1 \text { inv }} m_{1 \text { inv }}, \tau_{1 \text { inv }}, c_{1 \text { inv }}$, $\rho_{2 \text { inv }}$ - параметры, определенные в результате инверсии. Отношения $h_{1 \text { inv }} / h_{1}, \rho_{1 \text { inv }} / \rho_{1}, m_{1 \text { inv }} / m$, $\tau_{1 \text { inv }} / \tau, c_{1 \text { inv }} / c, \rho_{\text {2inv }} / \rho_{2}$ характеризуют относительные отклонения параметров, найденных путем инверсии, от истинных.

На рис. 4а показано, как в зависимости от мощности слоя изменяется отношение $h_{1 \mathrm{inv}} / h_{1}$. Для тонкого $\left(h_{1}<10 \mathrm{~m}\right)$ и мощного $\left(h_{1}>50 \mathrm{M}\right)$ слоев индивидуальная инверсия приводит к большим погрешностям в определении $h_{1}$. По данным совместной инверсии $h_{1 \mathrm{inv}}=h_{1}$ в интервале мощности слоя от 2м до 200м, т.е. при определении мощности слоя совместная инверсия дает несомненный положительный эффект.

Удельное электрическое сопротивление $\rho_{1}$ верхнего слоя по результатам индивидуальной инверсии определяется практически без погрешности при $h_{1} \geq 10 \mathrm{M}$ (рис. 4б). При малой мощности слоя совместная инверсия улучшает результат: уже при $h_{1}=2 \mathrm{M} \rho_{1 \text { inv }}$ отличается от $\rho_{1}$ не более чем на $(30-40) \%$.

При оценке поляризуемости слоя (рис. 4г) индивидуальная инверсия дает хороший или даже очень хороший результат, особенно для совмещённой установки. Совместная инверсия переводит хороший результат в ранг «идеального»: при любой мощности $h_{1}$ слоя $m_{1 \mathrm{inv}} / m_{1}=1$.

Что касается постоянной релаксации поляризационного процесса (рис. 4 д), то здесь - при $h_{1}=1$ м для установки «петля в петле» и при $h_{1}=200$ м для обеих установок - по данным индивидуальной инверсии отмечаются очень большие отклонения $\tau_{\text {inv }}$ от $\tau_{1}$. Совместная инверсия дает идеальный результат: при любой мощности слоя отношение $\tau_{1 \mathrm{inv}} / \tau_{1}=1$.

Показатель степени с (рис. 4е), определённый по результатам индивидуальной инверсии, в зависимости от установки и мощности слоя заметно отличается от истинного либо близок к нему. Неожиданным оказался тот факт, что отклонения $c_{1 \text { inv }} / c_{1}$ от единицы для совмещённой установки оказались больше по сравнению с теми, которые дала инверсия переходных характеристик установки «петля в петле». По данным совместной инверсии $c_{1 \mathrm{inv}}=c_{1}$ независимо от мощности верхнего слоя.

Удельное электрическое сопротивление основания, найденное путем индивидуальной инверсии $\left(\rho_{2 \text { inv }}\right)$, близко к $\rho_{2}$ при малой мощности слоя (рис. 4 в). Если $h_{1}$ превышает 10м (при инверсии данных совмещенной установки) и 50м (установка «петля в петле»), погрешности резко возрастают и $\rho_{\text {2inv }}$ отличается от $\rho_{2}$ почти на порядок. Совместная инверсия дает правильный результат во всем интервале $h_{1}$.

Таким образом, не располагая априорной информацией, интерпретатор правильно идентифицировал все модели как двухслойные с поляризующимся верхним слоем и неполяризующимся основанием. Напомним, что всего имелось 5 исходных моделей, для описания каждой из которых необходимо 6 параметров, а общее число подлежащих определению параметров равно 30. Отношение числа правильно определенных параметров к их общему числу логично использовать в качестве меры того, насколько успешной оказалась инверсия. Оказалось, что результаты индивидуальной инверсии оцениваются как положительные примерно в 65-70\% для каждой из установок. По данным совместной инверсии параметры модели удалось определить правильно в 90\% случаев, т. е. задача определения параметров модели могла считаться решённой.

\section{Отличия индукционно вызванной поляризации от наблюдаемой при работе с заземлёнными линиями}


Приведённое выше описание ВПИ как результат аддитивной «добавки» поляризационных токов к вихревым отражает близкую к реальной картину, если среда является слабо поляризующейся. В общем же случае имеет место единый процесс становления вихревых токов, контролируемый распределением удельной электропроводности, которая зависит от частоты/времени. При этом сохраняется фундаментальное свойство зондирований методом переходных процессов: чем больше временная задержка, на которой производится измерение ЭДС переходного процесса, тем больше эффективная глубина зондирования. Поэтому существует принципиальная и практическая возможность с помощью индукционных зондирований изучать распределение по глубине поляризуемости и других параметров (например, постоянной времени и показателя степени, если используется модель Коул-Коул).

Иногда возникают затруднения, связанные с необходимостью объяснить, почему при проведении съёмок методом ВП с заземлёнными линиями наблюдаются значительные аномалии поляризуемости, а проявления ВПИ отсутствуют, или же аномалии ВП и ВПИ не коррелируют [García-Fiscal, Flores, 2018; Macnae, Hine, 2016; Seidel, Tezkan, 2017]. На наш взгляд, это объясняется тем, что физика ВПИ отличается от той, на которой основан метод ВП.

В работе [Kozhevnikov, Antonov, 2018] рассмотрены процессы ВП, возбуждаемые с помощью источника тока и напряжения в образце, удельное электрическое сопротивление которого описывается формулой (1) при $c=1$ (дебаевская релаксация). В первом случае на образец воздействует ступень тока:

$I(t)=I_{0} \mathbf{1}(t), \quad(4)$

где $\mathbf{1}(t)$ - единичная функция Хевисайда, $I_{0}$ - амплитуда ступени.

В этом случае откликом на воздействие является переходный процесс напряжения:

$V(t)=I_{0} R_{0} \theta 1-m e^{-\frac{t}{\tau}}$,

где $R_{0}$-сопротивление образца на постоянном токе.

Из этого выражения видно, что: 1) при прочих равных условиях амплитуда отклика тем больше, чем больше $R_{0}$, т. е. чем выше сопротивление образца; 2) постоянная времени переходного процесса равна постоянной времени в формуле (1).

Если же на образец воздействует ступень напряжения с амплитудой $V_{0}$ :

$V(t)=U_{0} \mathbf{1}(t), \quad(6)$.

откликом является переходный процесс тока

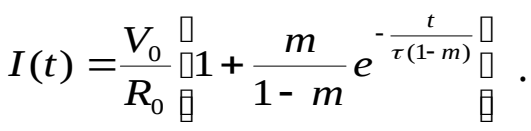

В этом случае время релаксации равно [составляет] $\tau(1-m)$, т. е. переходный процесс тока при воздействии на образец импульсом напряжения протекает быстрее, чем переходный процесс напряжения, когда воздействие осуществляется импульсом тока. Это находит отражение в методе переходных процессов. Как показано на рис. 4а в работе [...], чем выше 
поляризуемость, тем раньше - при прочих равных параметрах модели Коул-Коул проявляются эффекты ВПИ.

Напомним, что в традиционном методе ВП воздействие на среду осуществляется с помощью источника тока, а откликом является напряжение $V_{\text {вп }}(t)$ между электродами приёмной линии. При фиксированной поляризуемости $V_{\text {вп }}(t)$ тем больше, чем выше удельное электрическое сопротивление геологической среды или объекта. В индуктивных методах воздействием является электрическое поле, а откликом - плотность тока поляризации $j_{\text {вп }}(t)$, или - в терминах теории цепей - напряжение и ток [Wait, 1983]. Поэтому, чем ниже, при одной и той же поляризуемости, сопротивление среды или объекта, тем больше поляризационный ток. Это одна из причин, объясняющая, почему при изучении поляризующихся сред/объектов между результатами методов ВП и переходных процессов не всегда наблюдается корреляция.

Отметим также, что согласно выражению (7) при небольших значениях поляризуемости начальное значение тока поляризации возрастает прямо пропорционально $m$. Однако при $m \rightarrow 1$ начальное значение тока неограниченно возрастает, что приводит ко все более быстрому возрастанию интенсивности ВПИ. В качестве иллюстрации сошлемся на рис. 4 в нашей статье [Кожевников, Антонов, 2020].

Если возбуждение образца осуществляется с помощью источника напряжения, ток, протекающий через образец, создаваемое этим током магнитное поле и скорость его изменения зависят от $\sigma_{0}, m, \tau$ и с. Поэтому указанные параметры влияют на индукционные переходные характеристики и, соответственно, могут быть определены по результатам съёмок методом переходных процессов.

\section{Выводы}

Одним из эффектов последействия, присущих геологической среде и оказывающих влияние на результаты метода переходных процессов, является индукционно-вызванная электрическая поляризация (ВПИ). Её влияние на индукционные переходные характеристики выражается в нарушении монотонности спада ЭДС (включая изменение полярности).

Зависимость ВПИ от многих факторов затрудняет поиск и изучение основных закономерностей её проявления. Одним из путей решения этой проблемы является представление результатов моделирования с помощью нормированных переходных характеристик.

С наиболее общих позиций фактором, определяющим интенсивность и временной диапазон проявления ВПИ, является конкуренция между процессами индукции и вызванной поляризации.

В настоящее время не существует природных объектов, электрические свойства которых изучены настолько детально, чтобы на основе экспериментальных данных возможно было оценить качество инверсии данных МПП с учётом ВПИ. Поэтому большая роль отводится компьютерному эксперименту, спланированному так, чтобы как можно полнее имитировать реальные данные и процедуру их интерпретации.

Процессы вызванной поляризации протекают по-разному в зависимости от того, какой источник используется для их возбуждения. Поэтому при изучении поляризующихся сред не всегда наблюдается соответствие между результатами метода переходных процессов и традиционного метода ВП. 
Работа выполнена при поддержке проекта ФНИ № 0331-2019-0007 «Геоэлектрика в исследованиях геологической среды: технологии, полевой эксперимент и численные модели».

\section{ЛИТЕРАТУРА}

Антонов Е. Ю., Кожевников Н. О., Корсаков М. А. Автоматизированная система для интерпретации данных индукционных импульсных электромагнитных зондирований с учётом индукционно-вызванной поляризации // Геология и геофизика, 2014, т. 55 (8), с. $1282-1293$.

Кожевников Н. О., Антонов Е. Ю. Совместная инверсия данных МПП с учётом индукционновызванной поляризации // Геология и геофизика, 2009, т. 50 (2), с. 181-190.

Кожевников Н. О., Антонов Е. Ю. Инверсия индукционных переходных характеристик двухслойных сред с учётом быстро устанавливающейся вызванной поляризации // Геология и геофизика, 2010, т. 51(6), с. 905-918.

Кожевников Н. О., Антонов Е. Ю., Захаркин А. К., Корсаков М. А. Поиск таликов методом ЗСБ в условиях интенсивного проявления индукционно-вызванной поляризации // Геология и геофизика, 2014, т. 55 (12), с. 1815-1827.

Кожевников Н. О., Антонов Е. Ю. Индукционно-вызванная поляризация и оценка ее проявления с помощью нормированных переходных характеристик // Геология и геофизика, 2020.

Комаров В. А. Электроразведка методом вызванной поляризации. Л., Недра, 1980, 391 с.

Компаниец С. В., Кожевников Н. О., Антонов Е. Ю. Проявления и учёт индукционновызванной поляризации при изучении осадочного чехла юга Сибирской платформы методом ЗСБ // Геофизика, 2013, №1, с. 35-40.

Куликов А. В., Шемякин Е. А. Электроразведка фазовым методом вызванной поляризации: М., Недра, 1978, 157 с.

Левченко А. В. Взаимное влияние процессов индукции и вызванной поляризации при индуктивном и гальваническом возбуждении. Автореф. дисс. канд. техн. наук. Екатеринбург, 1992, 17 с.

Сидоров В. А. Импульсная индуктивная электроразведка. М., Недра, 1985, 192 с.

Стогний В. В. Импульсная индуктивная электроразведка при изучении поляризующейся среды криолитозоны Якутской кимберлитовой провинции // Криосфера Земли, 2008, т. XII (4), с. 46-56.

Эпов М. И., Антонов Е. Ю. Прямые задачи электромагнитных зондирований с учётом дисперсии геоэлектрических параметров // Физика Земли, 1999, № 3-4, с. А48-А55.

Эпов М. И., Антонов Е. Ю. Исследование влияния параметров вызванной поляризации при нестационарных электромагнитных зондированиях сложнопостроенных геологических сред // Геология и геофизика, 2000, т. 41 (6), с. 920-929.

Flis M. F., Newman G.A., Hohmann G.W., Induced polarization effects in time-domain electromagnetic measurements // Geophysics, 1989, v. 54, p. 514-523.

García-Fiscal S., Flores C., Sensitivity of galvanic and inductive Induced Polarization methods to the Cole-Cole parameters // Journal of Applied Geophysics, 2018, v. 158, p. 1-10.

Kamenetsky F.M., Trigubovich G.M., Chernyshev A.V. Three Lectures on Geological Medium Induced Polarization. Munich, Germany: Vela Verlag, 2014, 55 p.

Kang S., Fournier D., Oldenburg D.W. Inversion of airborne geophysics over the DO-27/DO-18 kimberlites - Part 3: Induced polarization // Interpretation, 2017, v. 5, p. T327-T340.

Kozhevnikov N.O., Antonov E.Y. Fast-decaying IP in frozen unconsolidated rocks and potentialities for its use in permafrost-related TEM studies // Geophysical Prospecting, 2006, v. 54, p. 383-397. 
Kozhevnikov N.O., Antonov E.Yu. Inversion of TEM data affected by fast-decaying induced polarization: Numerical simulation experiment with homogeneous half-space // Journal of Applied Geophysics, 2008, v. 66, p. 31-43.

Kozhevnikov N.O., Antonov E.Yu. Fast-decaying inductively induced polarization in frozen ground: A synthesis of results and models, Journal of Applied Geophysics, 2012, v. 82, p. 171-183.

Kozhevnikov N.O., Antonov E.Y. Current and voltage source induced polarization transients: a comparative consideration. Geophysical Prospecting, 2018, v. 66, p. 422-431.

Macnae J., Hine K., Comparing induced polarization responses from airborne inductive and galvanic ground systems: Tasmania // Geophysics, 2016, v. 81, p. E471-E479.

Marchant D., Haber E., Oldenburg D.W. Three-dimensional modeling of IP effects in time-domain electromagnetic data // Geophysics, 2014, v. 79, p. E303-E314.

Pelton W., Ward S., Hallof P., Sill W., Nelson P., Mineral discrimination and removal of inductive coupling with multifrequency IP // Geophysics, 1978, v.43, p. 588-609.

Raiche A.P. Negative transient voltage and magnetic field response for a half-space with a ColeCole impedance // Geophysics, 1983, v. 48, p. 790-791.

Seidel M., Tezkan B. 1D Cole-Cole inversion of TEM transients influenced by induced polarization // Journal of Applied Geophysics, 2017, v. 138, p. 220-232.

Seigel H., Nabighian M., Parasnis D.S., and Vozoff K. The early history of the induced polarization method // The Leading Edge, 2007, Vol. 26, no 3, 312-321.

Smith R.S., Klein J. A special circumstance of airborne induced polarization measurements // Geophysics, 1996, v. 61, p. 66-73.

Smith, R.S., Walker, P.W., Polzer, B.D., and West, G.F., 1988, The time-domain electromagnetic response of polarizable bodies: an approximate convolution algorithm, Geophysical Prospecting, 36, $772-785$.

Spies B.R. A field occurrence of sign reversals with the transient electromagnetic method // Geophysical Prospecting, 1980, v. 28, p. 620-632.

Sumner J.S. Principles of Induced Polarization for Geophysical Exploration. Amsterdam, Netherlands: Elsevier, 1976, 277 p.

Wait J.R., 1983. A simple view of the I.P. influence in an inductive E.M. prospecting system. IEEE Trans. Geosc. and Remote Sens. 21 (24), 505-506.

Walker G.G., Kawasaki K.K. Observation of double sign reversals in transient electromagnetic central induction soundings // Geoexploration, 1988, v. 25, p. 245-254.

Weidelt P. Response characteristics of coincident loop transient electromagnetic systems // Geophysics, 1982, v. 48, p. 1325-1330. 
Подрисуночные подписи к статье Н.О. Кожевникова, Е.Ю. Антонова «Эффекты последействия в методе переходных процессов: индукционно-вызванная поляризация».

Рис. 1. Немонотонные переходные процессы без изменения (ТЕМ 386) и с двойным изменением (ТЕМ 387, 388) полярности. Юго-западный фланг профиля 50-54-58, Накынское кимберлитовое поле, Якутия. Установка 200м х 100м.

Рис. 2. Влияние удельного электрического сопротивления на исходные (а) и нормированные б) переходные характеристики однородного полупространства. Шифр графиков - удельное электрическое сопротивление полупространства, Ом · м. Остальные параметры: $m=0.1, \tau=50$ мкс, $c=1$. Совмещённая установка размером 100м $\times 100 \mathrm{M}$.

Рис. 3. Зависимости $Y_{\min }\left(h_{1}\right)\left(\right.$ а) и $Y_{\min }\left(\rho_{1}\right)$ (б) для модели в виде поляризующегося $\left(m_{1}=0.1\right.$, $\tau_{1}=50$ мкс, $c_{1}=1$ слоя), перекрывающего неполяризующееся основание. Совмещённая установка размером $100 \mathrm{M} \times 100 \mathrm{M}$.

Рис. 4. Параметры, найденные в результате индивидуальной и совместной инверсии, в зависимости от мощности поляризующегося слоя: $h_{1 \mathrm{inv}}(\mathrm{a}), \rho_{\text {inv }}(б), \rho_{2 \mathrm{inv}}\left(\right.$ в), $m_{1 \mathrm{inv}}\left(\right.$ г), $\tau_{1 \mathrm{inv}}($ д), $c_{1 \text { inv }}(\mathrm{e})$. Инверсия выполнена одни из авторов статьи, который не располагал априорной информацией о моделях. 
Таблица 1. Влияние параметров однородного поляризующегося полупространства и размера генераторной петли на координаты минимума нормированной переходной характеристики и ширину временного диапазона, в котором проявляется ВПИ.

\begin{tabular}{|l|c|c|c|}
\hline \multicolumn{1}{|c|}{ параметр } & $t_{\min }$ & $Y_{\min }$ & $\begin{array}{c}\text { Временной диапазон } \\
\text { проявления ВПИ }\end{array}$ \\
\hline$m$ & - & - & + \\
\hline$\rho$ & - & - & - \\
\hline$\tau$ & + & - & - \\
\hline$c(>0.5)$ & - & - & + \\
\hline $\begin{array}{l}\text { Длина стороны } \\
\text { генераторной петли }\end{array}$ & + & + & + \\
\hline
\end{tabular}


Таблица 2. Параметры исходных моделей с поляризующимся верхним слоем и неполяризующимся основанием.

\begin{tabular}{|l|l|l|l|l|l|l|}
\hline $\mathrm{N}$ & $h_{1}, \mathrm{M}$ & $\rho_{1}, \mathrm{OM} \cdot \mathrm{M}$ & $m_{1}$ & $\tau_{1}, \mathrm{C}$ & $c_{1}$ & $\rho_{2}, \mathrm{OM} \cdot \mathrm{M}$ \\
\hline 1 & 1 & 100 & 0.1 & $5 \cdot 10^{-5}$ & 1 & 1000 \\
\hline 2 & 2 & 200 & 0.1 & $5 \cdot 10^{-5}$ & 0.9 & 500 \\
\hline 3 & 10 & 100 & 0.05 & $5 \cdot 10^{-5}$ & 1 & 1000 \\
\hline 4 & 50 & 50 & 0.2 & $1 \cdot 10^{-4}$ & 0.95 & 2000 \\
\hline 5 & 200 & 200 & 0.1 & $2.5 \cdot 10^{-5}$ & 0.9 & 1000 \\
\hline
\end{tabular}




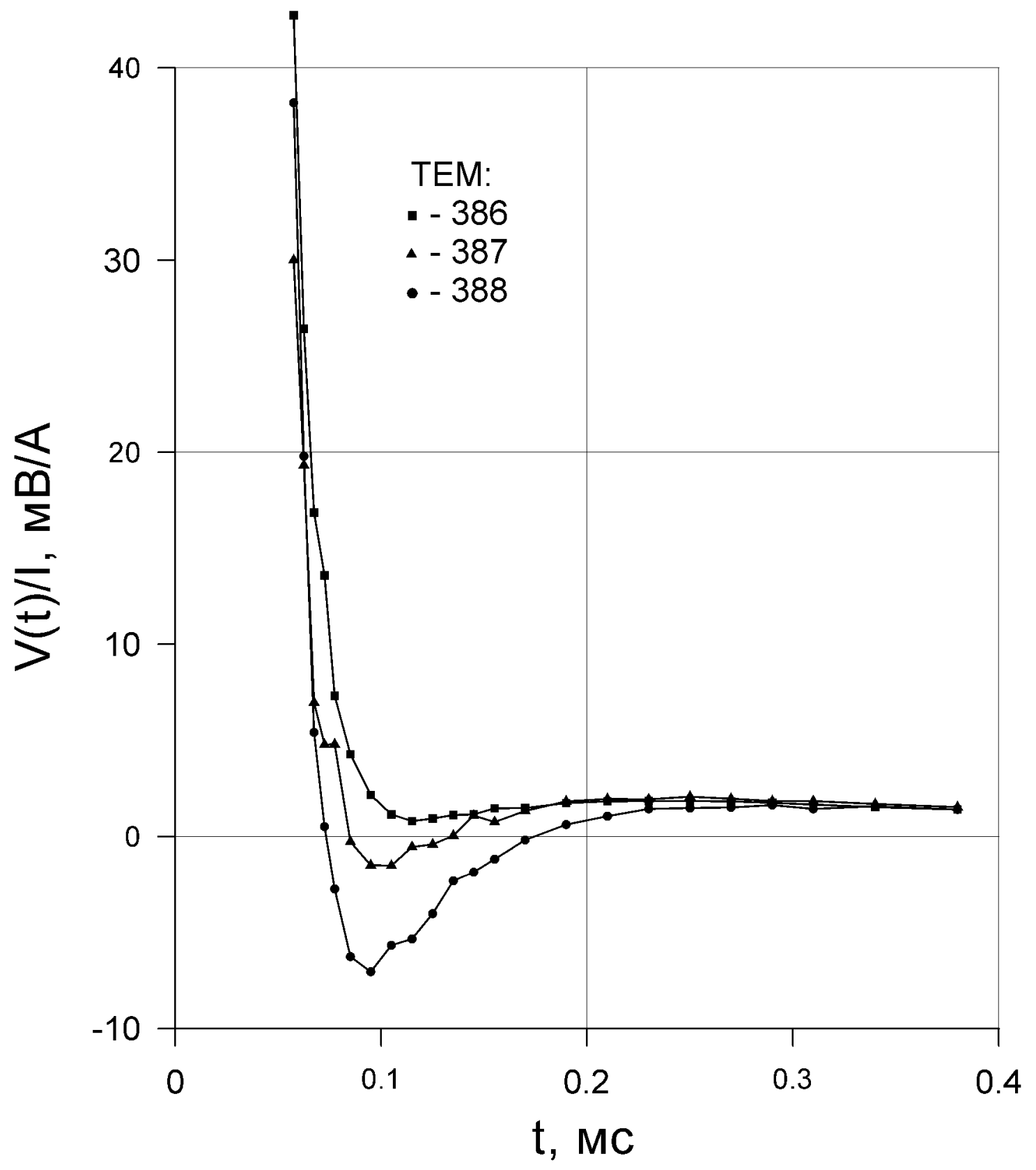


a)

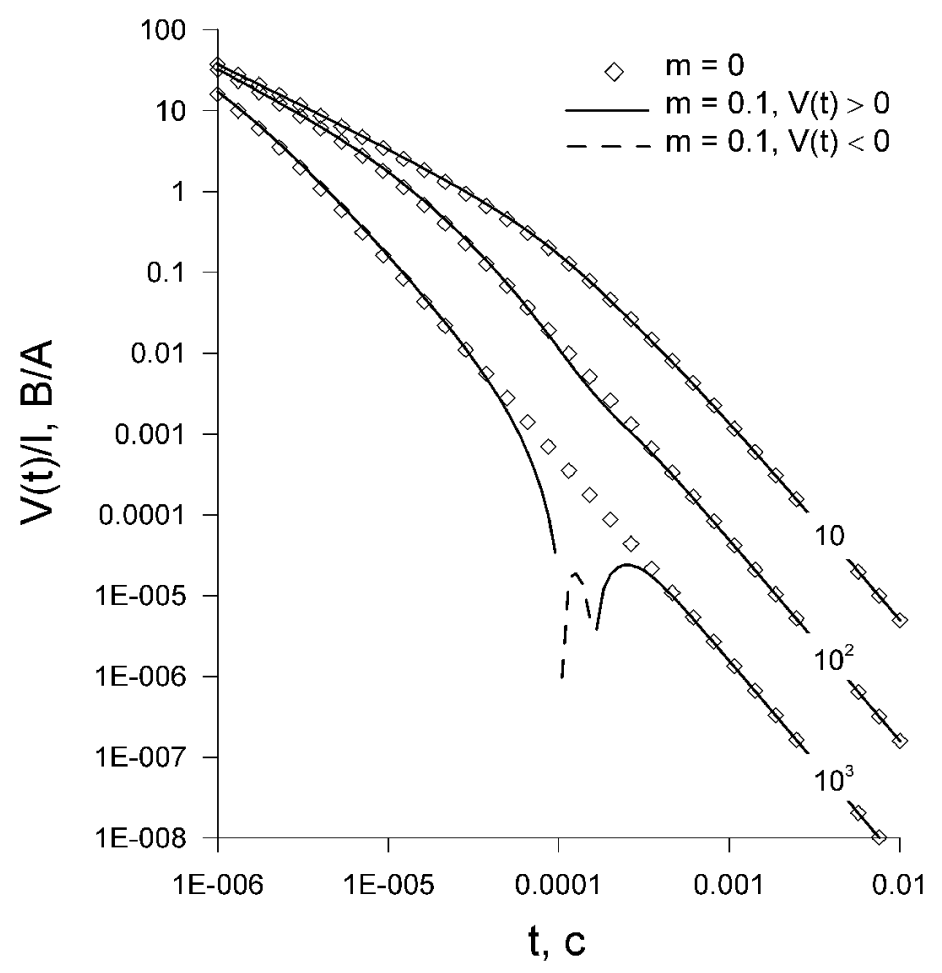

б)

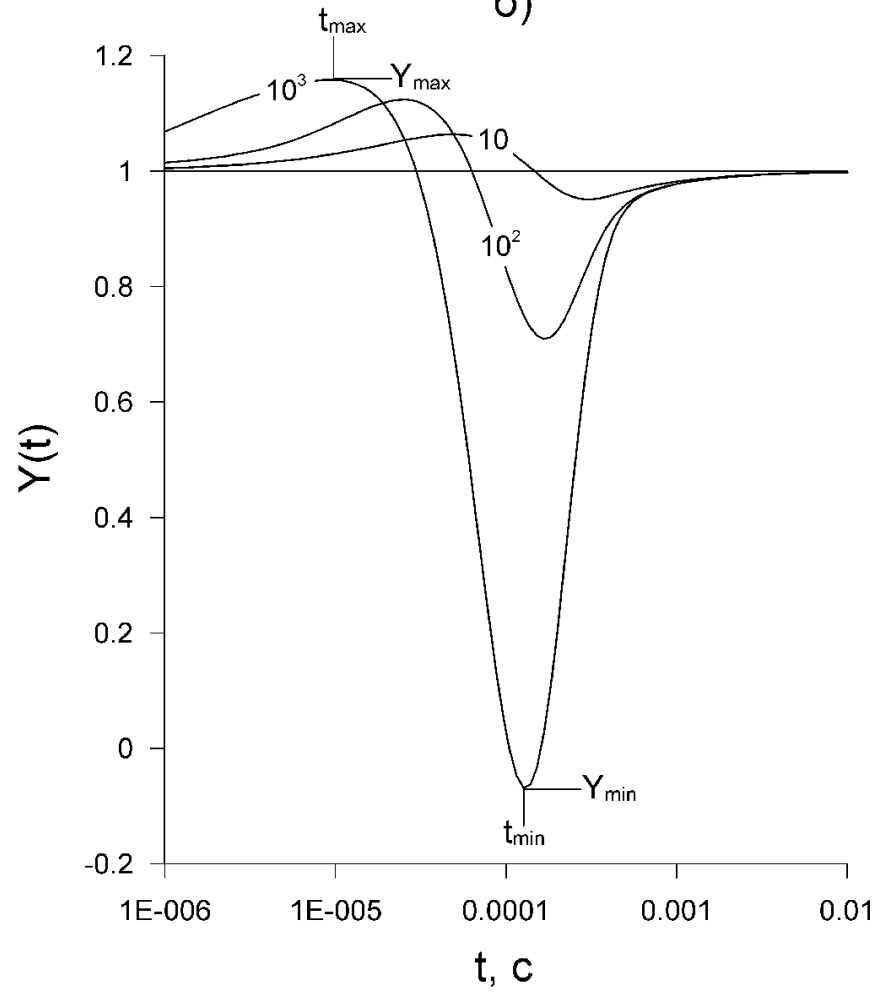


a)

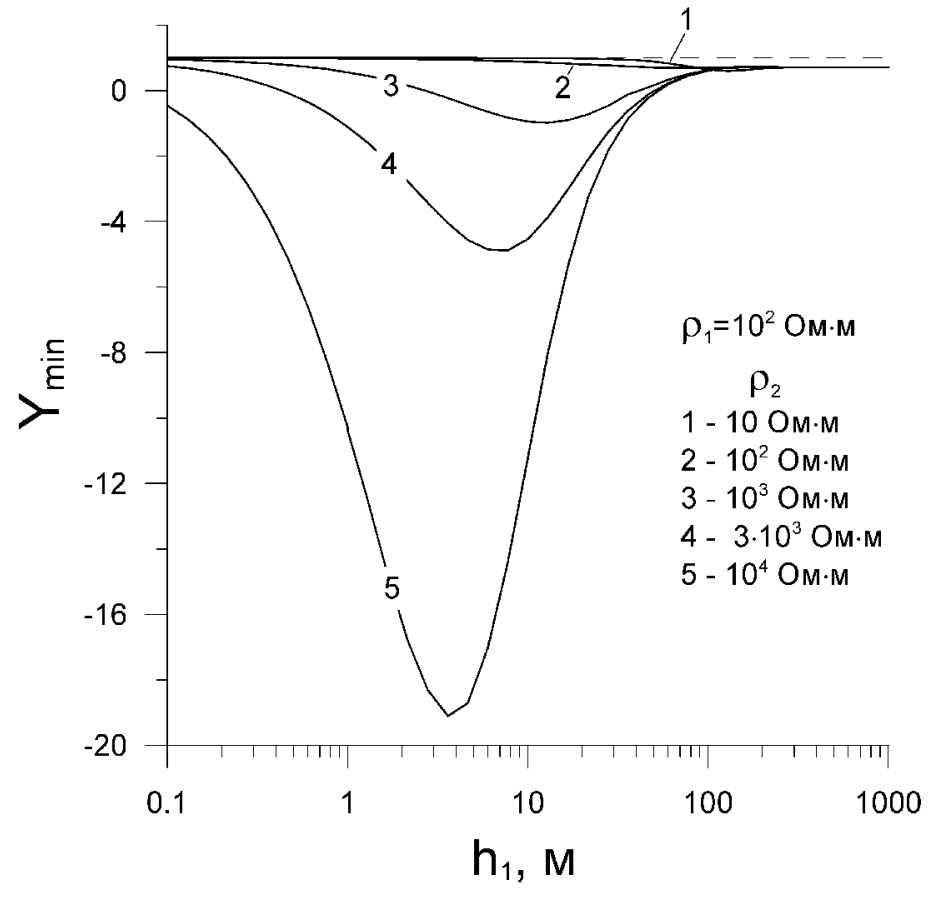

б)

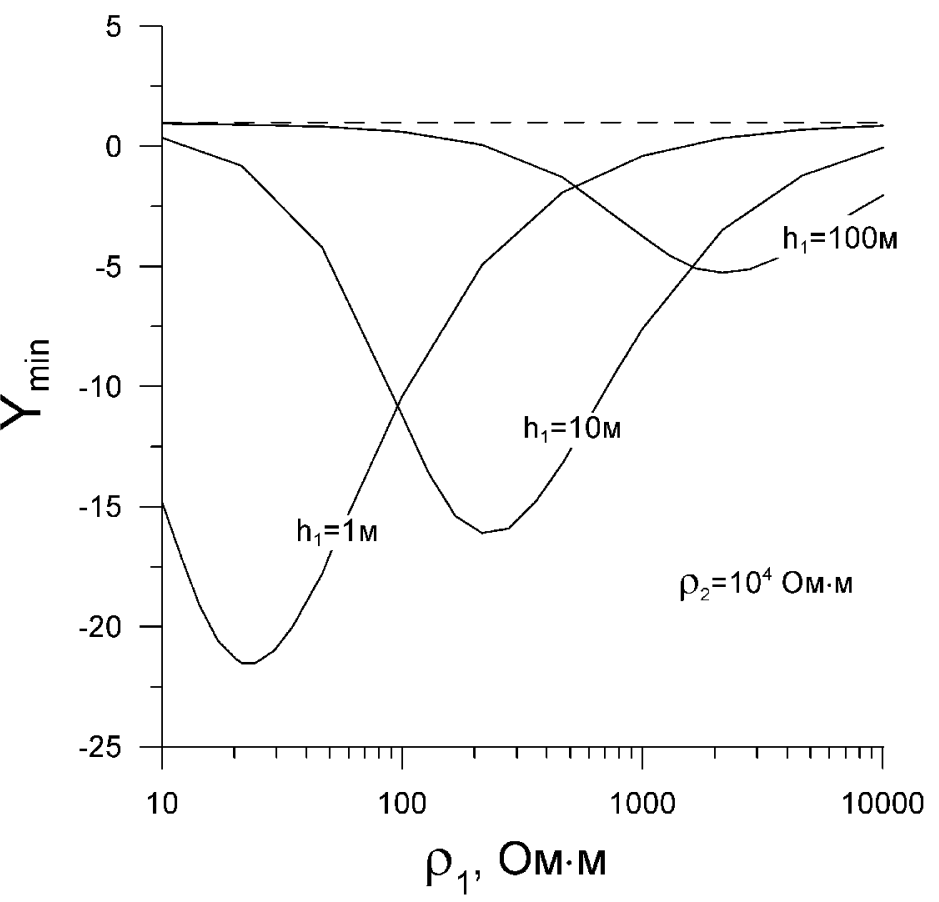



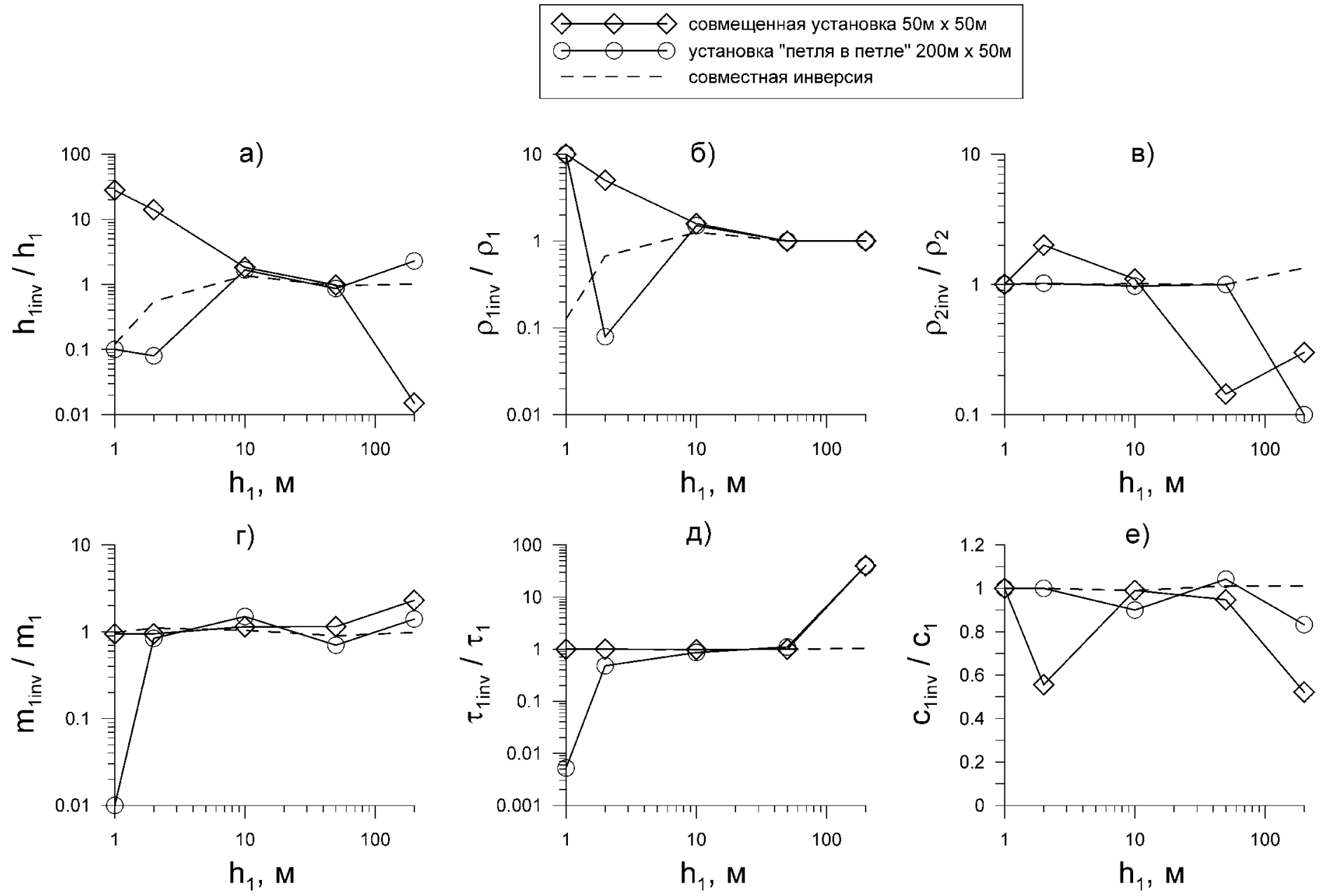